\title{
Geometric Factors in Electrical Measurements Relating to Corrosion and Its Prevention
}

\author{
W. J. Schwerdtfeger and Irving A. Denison
}

\begin{abstract}
The "electrical boundary" of a galvanic couple immersed in an aqueous medium, when corroding normally and also when corrosion is stopped by cathodic protection, is discussed in the light of present theory. Experimental data, consisting of potential measurements made on bimetallic couples in an unrestricted medium producing relatively little polarization and also one producing considerable polarization, are compared with theoretical data. The electrical boundary is theoretically defined and experimentally verified for model galvanic couples having bimetallic electrodes, one couple simulating line electrodes and the others having electrodes made of longitudinal cylindrical segments arranged in three anode-tocathode area ratios. The effect on potential measurements as a result of interference to the normal flow of galvanic and external currents is also shown by experimental data. The results of this study indicate that geometric factors should be given consideration in making potential measurements on subsurface structures of similar configuration, such as cylindirical tanks and pipelines.
\end{abstract}

\section{Introduction}

Because of the electrochemical nature of corrosion in aqueous media, the study of current and potential relations pertaining to galvanic couples has provided much useful information concerning the mechanism of corrosion and its prevention. However, relatively little attention has been given to the spacial relations between the anodic and cathodic areas that comprise the galvanic couples. In a given medium, potentials ${ }^{1}$ are affected by the polarization of the elements of the couple and also by the conductivity of the electrolyte. In order to study the potential pattern around a galvanic couple ${ }^{2}$ most effectively, measurements might be made with the couple in a solution which causes relatively little polarization. The conclusions reached from these measurements, insofar as geometrical considerations are concerned, should also be applicable to all galvanic couples of similar configuration, regardless of polarization or electrolyte conductivity. It might even be true that in certain environments such geometrical considerations become relatively insignificant, for example, in a highly conductive electrolyte producing considerable polarization.

The plan of this investigation was to make an experimental study, based on theory, of the potential distribution in the vicinity of line electrodes immersed in a conducting medium and to show the relation between the potential pattern and geometry. The relationship was then extended to experimental galvanic couples in the form of cylinders, with the aim of perhaps applying the information obtained to the measurement of potentials on subsurface structures of similar configuration, such as pipelines.

\footnotetext{
1 The term "potential" as used in this faper implies a difference of potential between a standard reference electrode and the element under discussion in the same electrolyte. When current flows, resistance drop is included.

2 The term "galvanic couple" implies the flow of current produced locally by cell action due to the contact of dissimilar metals in a certain environment or because of the environmental effect on different areas of the surface of a metal.
}

\section{Theoretical Considerations}

\subsection{Definition of Symbols}

The electrical symbols used throughout this paper are identified and defined as follows:

$E_{a}=$ Open-circuit potential of the anode

$E_{c}=$ Open-circuit potential of the cathode.

$E_{g}=$ Potential of the couple.

$\Delta E_{a}=$ Change in anode potential.

$\Delta E_{c}=$ Change in cathode potential.

$E_{p}=$ Potential at some arbitrary point $P$.

$E_{t}=$ External voltage applied to the couple.

$E=$ Potential at the electrical boundary of the couple when external current is cathodically applied. For the experimental data in this paper, $E$ is associated with the current $I_{p}$.

$e_{a}=$ Anode (surface) driving potential.

$e_{c}=$ Cathode (surface) driving potential.

$e_{c}^{\prime}=$ Potential of the cathode during cathodic protection.

$I=$ External current to the couple.

$I_{p}=$ External cathodic current flowing to the couple when the cell current $i_{0}=0$, defined as the current necessary for cathodic protection.

$i_{0}=$ Cell current

$R_{g}=$ Resistance of the couple to the flow of external current.

$r_{a}=$ Electrolytic anode resistance.

$r_{c}=$ Electrolytic cathode resistance.

$r_{0}=r_{a}+r_{c}=$ Electrolytic cell resistance.

$r_{s}=$ Electrolytic resistance external to the electrical boundary.

\subsection{Galvanic Couple Without External Current}

When the anode and cathode of a galvanic couple do not polarize the following relation applies:

$$
E_{c}-E_{a}-i_{0} r_{0}=0 \text {. }
$$

If the anode and cathode do polarize and the cell resistance $r_{0}$ is expressed in terms of its anodic and cathodic components, eq (1) becomes

or

$$
e_{c}-e_{a}-i_{0} r_{a}-i_{0} r_{c}=0
$$

$$
e_{a}+i_{0} r_{a}=e_{c}-i_{0} r_{c}=E_{g} .
$$


Equation(3) is pictured diagrammatically in figure 1. If a reference electrode is dipped into the electrolyte in the vicinity of the couple and moved from the anode surface to the cathode surface, the measured potential, $E_{p}$, between the reference electrode and the couple will vary from $e_{a}$ through $E_{g}$ to $e_{c}$. If the reference electrode is sufficiently far removed from the couple so as to be out of the influence of the anode and cathode, the potential measured will be $E_{g}$ in all positions. The variation in potentials observed will depend chiefly on the difference between the open-circuit potentials, $E_{a}$ and $E_{c}$, and the polarization, $\Delta E_{a}$ and $\Delta E_{c}$, after the couple is formed.

A better understanding of the couple potential is possible if one imagines two line electrodes, $A$ and $C$, of infinite length surrounded by an unbounded electrolyte, as shown in figure 2 , a, disregarding the circle for the present. If a current is flowing between anode $A$ and cathode $C$, the potential, $E_{p}$, at some point, $P$, is expressed spatially by the function $\log \rho_{2} / \rho_{1}$, as illustrated in figure $2, \mathrm{~b}[1]^{3}$, where $\rho_{1}$ and $\rho_{2}$ are, respectively, the distances between the anode and the cathode and point $P$. If, for example, the line electrodes are separated by a distance of 2.25 inches, the potential variation along the $A A^{\prime}$ axis with respect to the intersection of the $A A^{\prime}$ and $B B^{\prime}$ axes would be as shown in figure $2, b$. It will be noted that the potential, $E_{g}$, is approached asymptotically by values measured along the $A A^{\prime}$ axis, as indicated, or on all axes other than $B B^{\prime}$, which is an equipotential axis equal to the potential of the couple. For the nonpolarized line electrodes shown,

$$
E_{g}=\frac{E_{a}+E_{c}}{2}
$$

For a condition of polarized line electrodes, the expression becomes

$$
E_{g}=\frac{e_{a}+e_{c}}{2}
$$

${ }^{3}$ Figures in brackets indicate the literature references at the end of this paper.

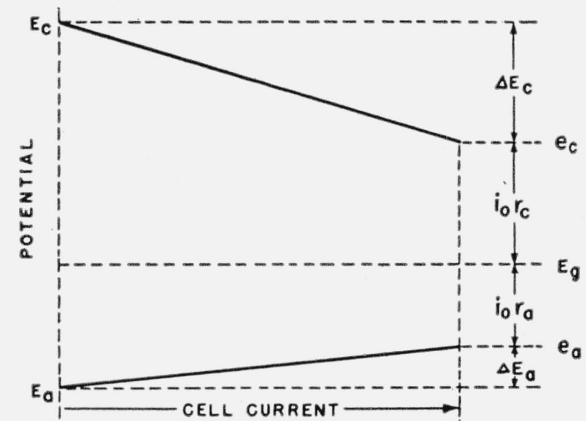

Figure 1. Electrical relations in a galvanic couple.
If the couple elements are no longer in the form of line electrodes, the couple potential, $E_{g}$, in relation to $e_{a}$ and $e_{s}$ will depend on the relative values of $r_{a}$ and $r_{c}$ (fig. 1).

\subsection{External Current Applied to a Cylindrical Conductor}

If an equipotential cylindrical surface of infinite length (fig. 2,a), disregarding the couple $A C$, is surrounded by an unbounded electrolyte, the electrical resistance of the cylinder to the radial flow of current is expressed geometrically by the function $\log D / a$, where $D$ and $a$ are both in the same units, $D$ being the radius of a concentric circle and $a$ the radius of the cylindrical conductor [2]. Therefore, if the conductor is subject to a radial flow of direct current, it will be surrounded by concentric equipotential circles and the potential, $E_{p}$, along any radius will vary as the function $\log D / a$, assuming that the current is constant and the potential of the conductor does not change during the course of the measurement. It will be shown (experimentally) later that this approximates the potential distribution at some distance away from a galvanic couple in the form of a cylinder under the condition of an externally applied current.

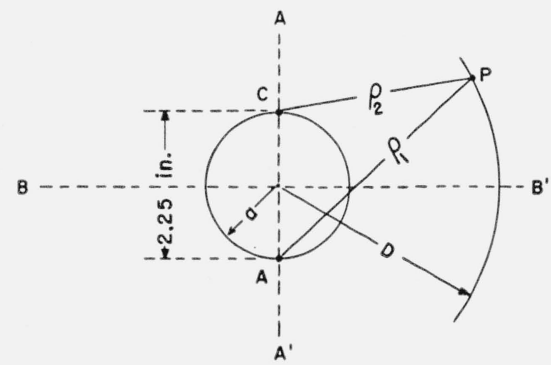

(a)

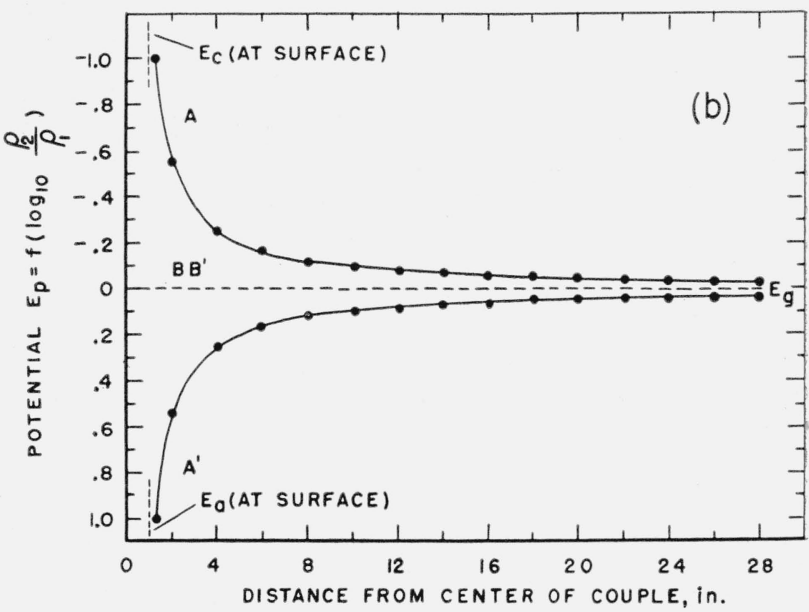

FIGURE 2. Theoretical potential of nonpolarized line electrodes. 


\subsection{Effective Electrical Boundary}

It has been shown that the theoretical potential of a couple is not reached within finite distance, except along the $B B^{\prime}$ axis. Actually, however, in a given electrolyte, the distance away from the couple where changes in potential, $E_{p}$, become insignificant is considered as the electrical boundary [3], and the corresponding potential is looked upon as the potential, $E_{g}$, of the couple.

It is sometimes desirable to measure the potential of a galvanic couple while an external current is flowing to it. Under such circumstances the electrical boundary, as previously defined, becomes obscured because of the $I R$ drop resulting from the applied current. A simultaneous evaluation of both the local and external current effects might be imagined if one considers the couple and the cylinder (fig. 2,a). In figure 3 are shown the logarithmic potential functions $\log _{10} \rho_{2} / \rho_{1}$ and $\log _{10} D / a$ for the couple and cylinder (diameter, 2.25 inches), respectively, plotted against the distance from the intersection of the $A A^{\prime}$ and $B B^{\prime}$ axes (center of the couple) as the abscissa. Also, if the abscissa were expressed as multiples of the distance $A C$ (fig. 2, a), the resulting curves would be similar to those of figure 3 .

It will be observed that beyond, for example, 14 inches (abscissa) the change in the couple potential function $\log \rho_{2} / \rho_{1}$ becomes relatively small in comparison with the change in the cylindrical potential function $\log D / a$. Therefore, if external current flows to the cylinder with the superimposed couple actually in direct contact, the concentric equipotential lines previously discussed in connection with the cylinder only would be expected, for all practical

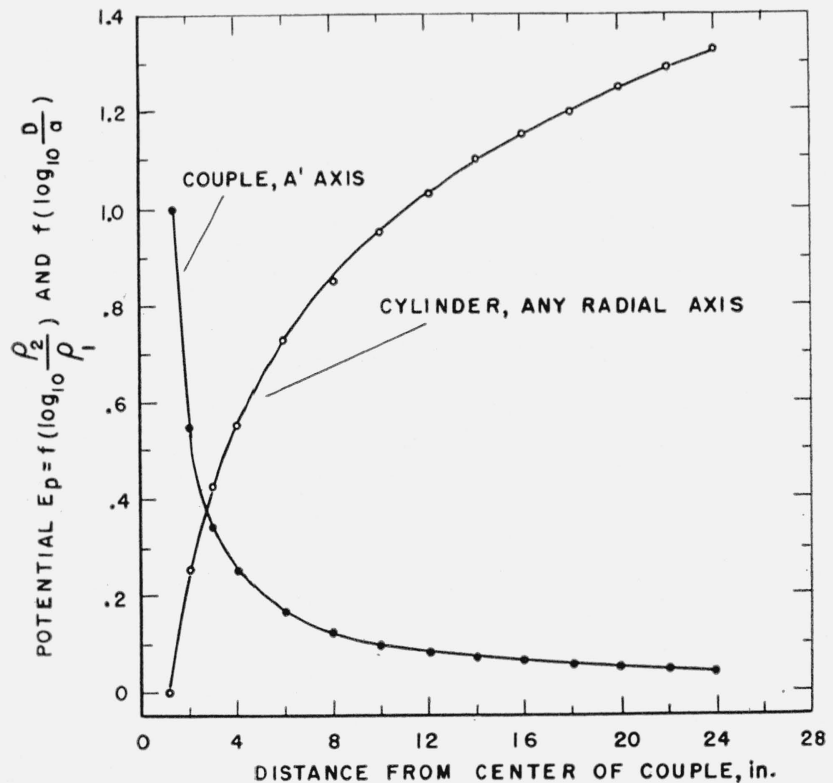

Figure 3. Comparison of the potential of a galvanic couple without external current and the potential around a cylinder receiving current.

Data apply for the dimensions in figure 2 (a) purposes, to still exist beyond the electrical boundary. The limiting distance of 14 inches, geometrically expressed, is about 6 diameters and might be looked upon as the "effective electrical boundary" of a galvanic couple diametrically superimposed on and in contact with a conducting cylinder of infinite length in an unrestricted electrolytic medium.

The accuracy of the geometric factor 6 as related to the effective electrical boundary can be experimentally evaluated by comparing the potential measured at 6 diameters on a laboratory model with the calculated boundary potential, $E$, pertaining to the model, based on the work of Holler $[4,5]$. Holler has shown that the resistance, $R_{g}$, of a galvanic couple, is

$$
R_{g}=\frac{r_{a} r_{c}}{r_{a}+r_{c}}
$$

Thus in figure 4, when the switch is closed, the following equations apply:

Let,

$$
E_{t}-I r_{s}-\left(E_{g}-I R_{g}\right)=0 .
$$

Then,

$$
E=E_{t}-I r_{s} .
$$

$$
E=E_{g}-I R_{g},
$$

the potential at the electrical boundary.

When the applied current, $I$, is equal to $I_{p}$ (see definition of symbols), the potential of the anode, $A$ will be $E_{a}$ because no current is leaving the anode. When $i_{0}=0$, then eq (3) becomes

$$
E_{u}=e_{a}=E_{a}
$$

and eq (9) becomes

$$
E=E_{a}-I_{p} R_{g}
$$

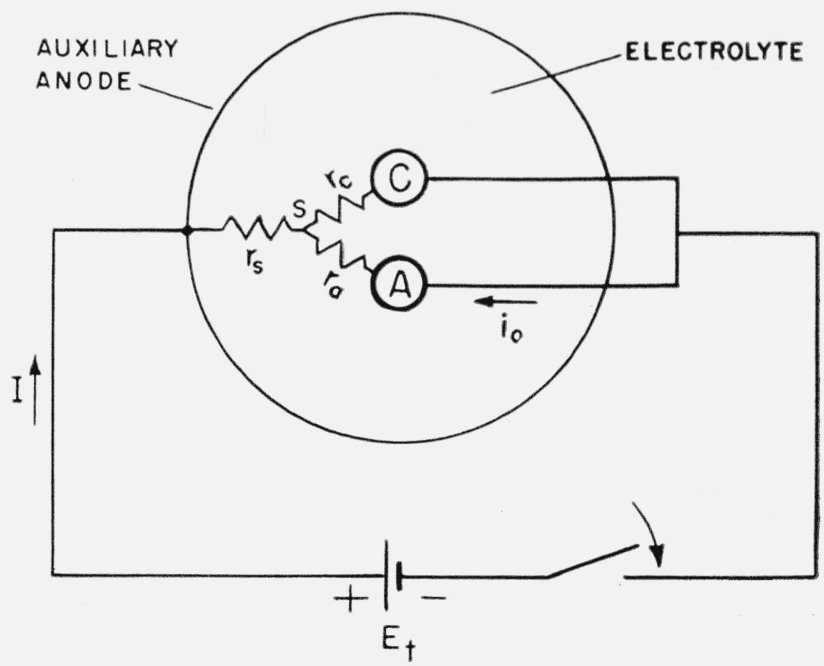

Figure 4. External current applied to a galvanic couple. 
$E_{a}$ and $I_{p}$ are measurable, and $R_{g}$ can be calculated. In an electrolyte producing relatively little polarization of the electrodes, $A$ and $C$, after measuring the potential, $E_{\mathrm{g}}$, the electrode potentials, $e_{a}$ and $e_{c}$, can also be measured by placing the reference electrode adjacent to the anode and cathode surfaces, respectively. The galvanic, or cell, current, $i_{0}$, can be measured with a zero-resistance milliammeter. From eq (3) and figure 1, it is apparent that

$$
i_{0} r_{a}=E_{\mathrm{g}}-e_{a} \text { and } i_{0} r_{c}=e_{c}-E_{g}
$$

therefore,

$$
r_{a}=\frac{E_{g}-e_{a}}{i_{0}} \text {, and } r_{c}=\frac{e_{c}-E_{g} .}{i_{0}}
$$

The value $R_{g}$ can now be calculated, and by substituting appropriate values in eq (11), the calculated potential, $E$, at the electrical boundary might be compared with the value actually measured with the reference electrode supposedly at the boundary.

\section{Experimental Results}

\subsection{Laboratory Methods}

The measurements were made in a cylindrical wooden vat approximately 66 inches in inside diameter and 18 inches high. The inner wall of the vat was lined with a copper sheet, which served as the auxiliary anode when external current was applied to the experimental cathodes. A $3 / 8$-inch steel rod was rigidly mounted off-center across the top of the open vat to hold $\mathrm{C}$-clamps for securing the galvanic couples in the center of the tank perpendicular to the wooden bottom. The rod also facilitated fastening and adjustment of the probe electrode for measurements made along the $A A^{\prime}$ axis. All potentials were measured with a high-resistance voltmeter $(200,000$ ohms/volt) and a saturated calomel half-cell terminated in a 8-foot-long flexible plastic tube and probe electrode, both of which were filled with a saturated potassium chloride-agar mixture. All potential measurements were made in a horizontal plane about 2 inches below the surface of the electrolyte in the vat. The electrolyte was maintained in level to a depth about 1 inch from the top of the vat.

\subsection{Galvanic Couple of Simulated Line Electrodes in a Dilute Copper Sulfate Solution}

The first experiments consisted of observations on galvanic couples having elements of zinc and copper in the form of paired rods of varying diameter in a solution of approximately $N / 10$ copper sulfate. Small-diameter rods ( 0.125 inch) were the nearest approach to theoretical line electrodes, but it was found that instability prevailed because of polarization due to the large galvanic-current densities, when such rods were coupled. However, rods of $5 / 16$-inch diameter or greater were polarized relatively little

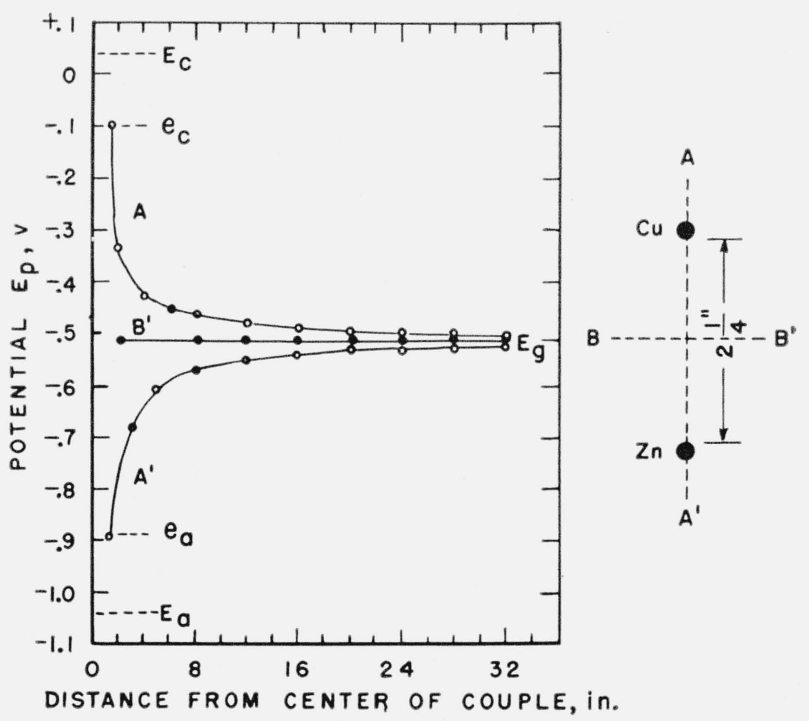

Figure 5. G'alvanic couple of line electrodes in a one-tenth normal copper sulfate solution.

$i_{0}=185 \mathrm{ma}$.

in the solution and therefore were free from unstable surface effects. Data obtained on a pair of externally short-circuited 5/16-inch zinc and copper rods are shown in figure 5 . The rods were separated by 2.25 inches to allow for the insertion later of a 2.25 -inchoutside-diameter conducting cylinder between the rods so that the data obtained might be compared with the theoretical diagrams (figs. 2 and 3 ). It will be noted that 90 percent of the change in potential, attributable to $I R$ drop, between the cathode and anode surface potentials, $e_{c}$ and $e_{a}$, respectively, and the couple potential, $E_{g}$, occurs at 10 inches in either direction along the $A A^{\prime}$ axis and the intersection with the $B B^{\prime}$ axis, namely, the center of the couple. This agrees quite well with the theoretical data, figure 2,b. Actually, if the electrodes are to be considered strictly as line electrodes, it would probably be more appropriate to consider their separation as being equal to the distance between their centers, namely, 2.56 inches.

Figure 6 shows potentials measured along the $A$ and $A^{\prime}$ axes for the same couple, potentials along the $B B^{\prime}$ axis being omitted, for the condition when the galvanic current $i_{n}=0$, accomplished by applying external current, $I_{p}$. The potential, $E$, at the electrical boundary calculated by the use of eq (11), is shown as one of the dashed horizontal lines in the figure crossing the $A^{\prime}$ and $A$ curves at 12 and 14 inches, respectively, and therefore is in fair agreement with the geometric electrical boundary (6 times distance of separation).

In figure 7 is shown the effect obtained by inserting a conducting cylinder (steel tube) between the copper and zinc electrodes (fig. 5), the copper rod being soldered to the steel. In comparing figures 5 and 7 it will be observed that, as a result, the couple 


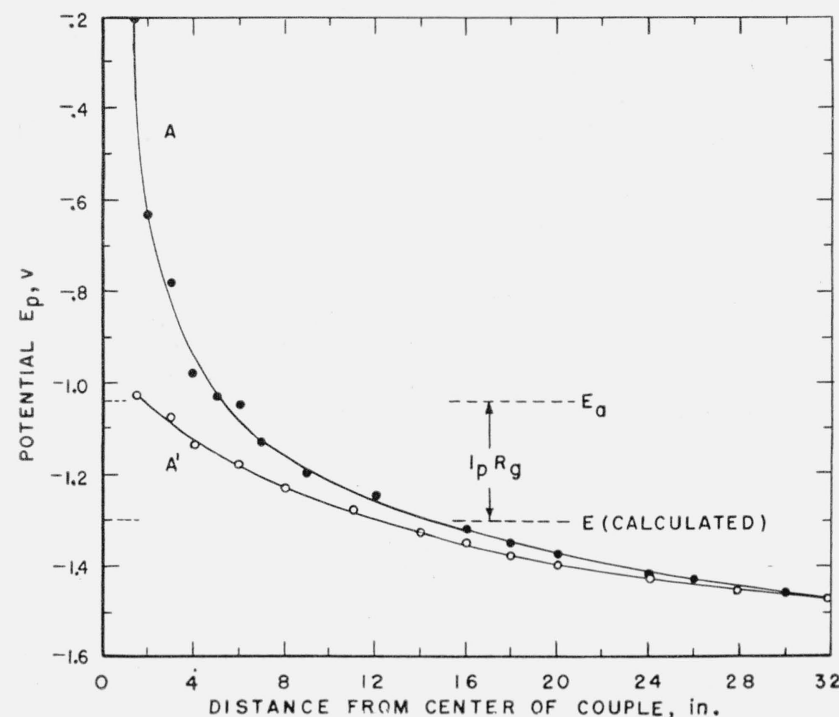

Figure 6. External cathodic current applied to the couple (fig. 5).

$I_{p}=230 \mathrm{ma}, i_{0}=0$.

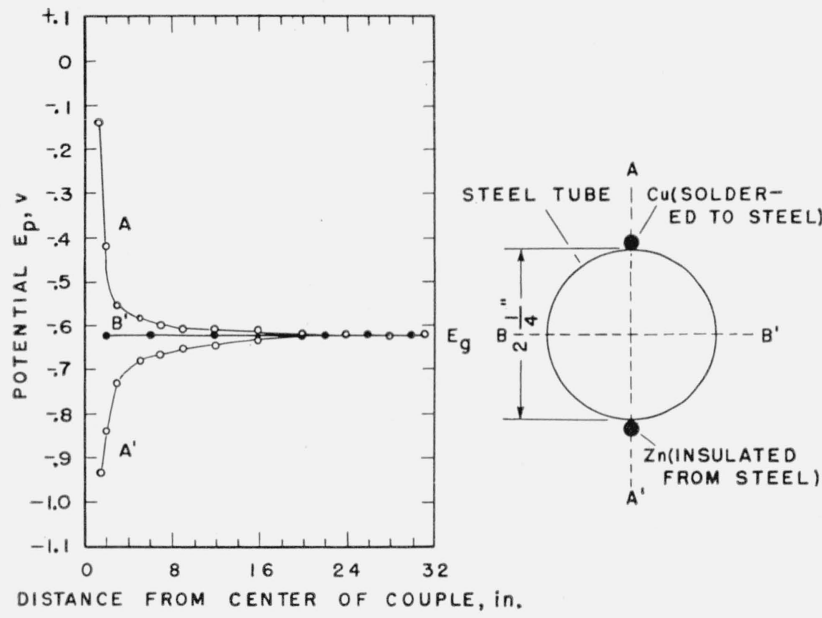

FIgURE 7. Same as figure 5, except for the steel tube. $i_{0}($ between $\mathrm{Zn}$ and $\mathrm{Fe}-\mathrm{Cu})=170 \mathrm{ma}$

potential, $E_{g}$, changed, but the effective electrical boundary of the $\mathrm{Zn}-\mathrm{Cu}$ couple remained about the same. When external current was applied (fig. 8) the effect of the copper and the zinc became negligible only after 14 inches, showing that the copper electrode determined the electrical boundary, even though the steel was the larger cathodic element of the couple. Although not pertinent to the present discussion, but important from the standpoint of cathodic protection, is the fact that $750 \mathrm{ma}$, as compared to 230 ma (fig. 6), had to be applied from the external power source in order to reduce the galvanic current to zero. This was so because the inclusion of the steel, having an open-circuit potential of $-0.61 \mathrm{v}$, put the system of galvanic couples under anodic control.

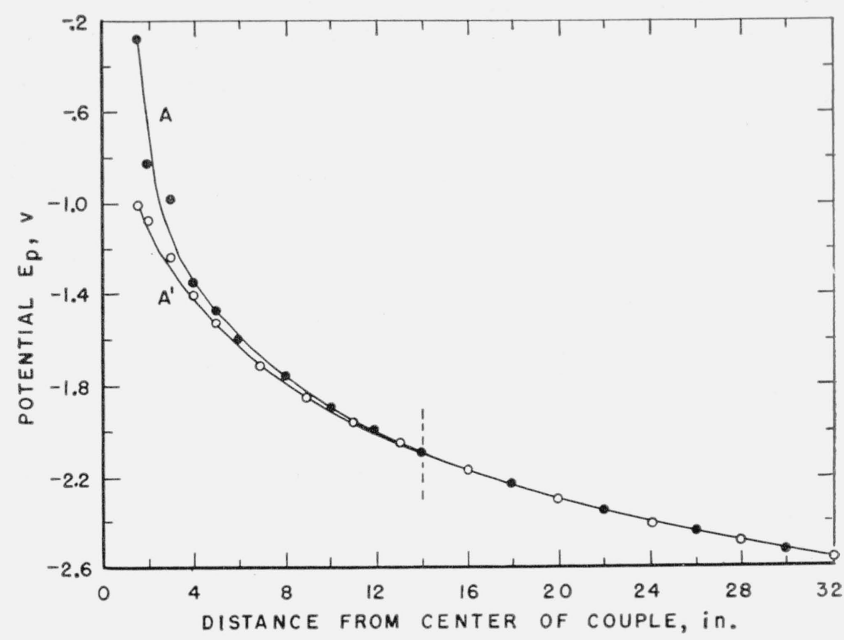

FIGURE 8. External cathodic current applied to the combination of couples (fig. \%).

$I_{p}=750 \mathrm{ma}, i_{0}=0$.

\subsection{Galvanic Couples With Electrodes Consisting of Cylindrical Segments in the Dilute Copper Sulfate Solution}

In order to determine whether the potential distribution around galvanic couples in the form of cylinders was similar to that in the vicinity of line electrodes, model cylindrical couples were constructed having the same diameter as the distance between the line electrodes discussed in the previous experiments.

The combination of a galvanic couple and conducting cylindrical surface might be represented by a cylinder having two longitudinal segments of different metals. The experimental models with which the remaining data were obtained consisted of such bimetallic cylinders having three area ratios of steel and copper with insulation between the metals to enable the measurement of galvanic current. Only the exterior cylindrical surfaces were exposed to the electrolyte.

Data obtained on the three cylindrical models are plotted in figures 9,10 , and 11 . In addition to potentials on the $A A^{\prime}$ and $B B^{\prime}$ axes, are shown also potentials measured along intermediate axes $C C^{\prime}$ and $D D^{\prime}$. Axis orientation is shown in the inset. part (a) of the figures. The change in potential along the $A A^{\prime}$ axis (fig. 9, a) compares on a percentage basis with the theoretical change for line electrodes (fig. 2,b). Calculated boundary potentials, $E$, eq (11), are indicated in parts (b), shown as covering a range in number of model diameters (1 diam, 2.25 in.) along the abscissas. For these models the boundaries might be imagined as resulting from composite couples in the form of paired line electrodes. The electrical boundary obtained by averaging the ranges resulting from the three sets of calculated data (figs. 9, 10, and 11) is about 3 diameters (based on model diameter).

The data in parts (c) of figures 9, 10, and 11 were obtained in order to compare potentials, $E_{p}$, of the 


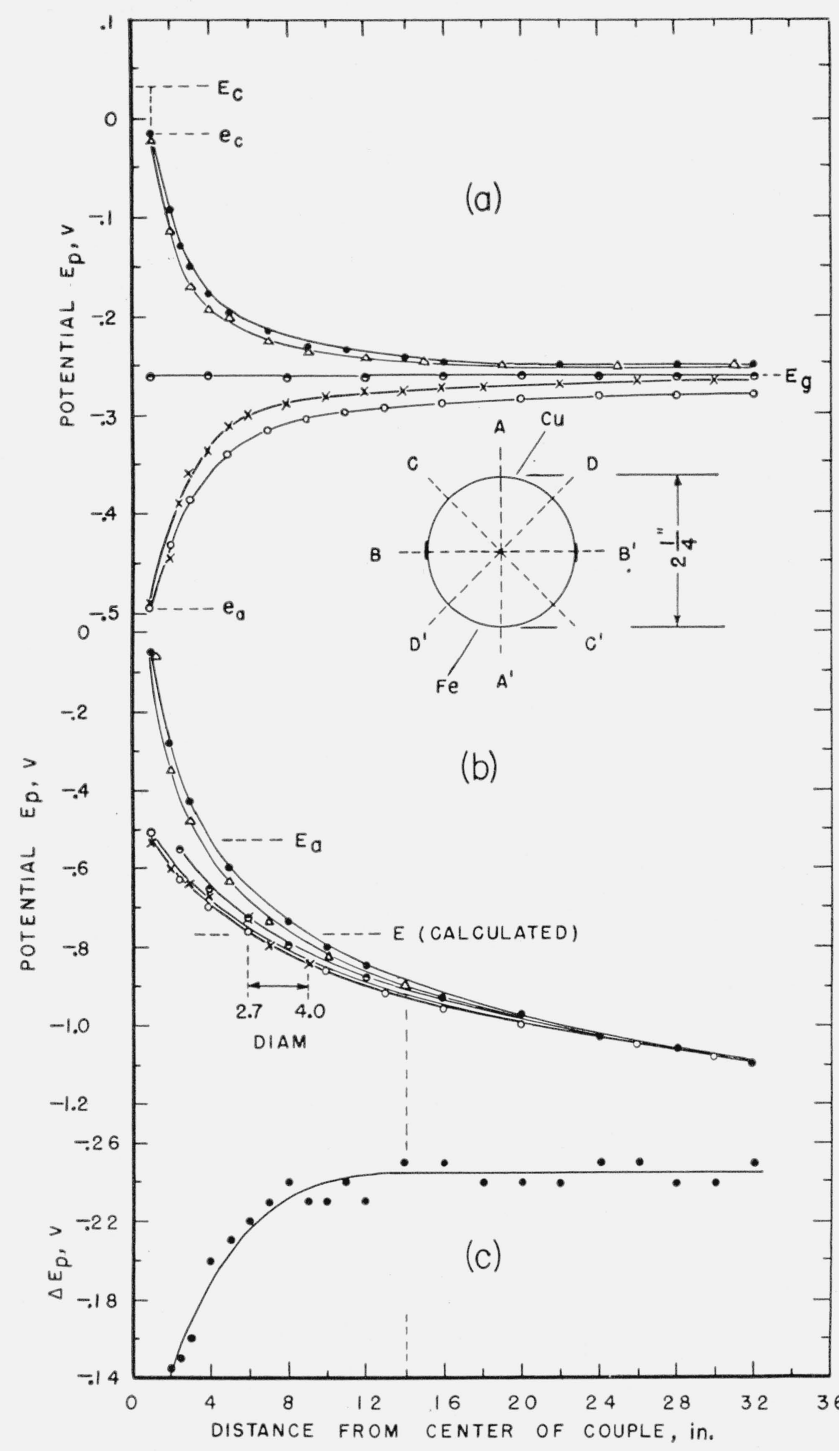

FIgure 9. Cylindrical galvanic couple in a one-tenth normal copper sulfate solution.

'. Area ratio, $\mathrm{Fe}: \mathrm{Cu}=1: 1$. $A$ axis; $\bigcirc, A^{\prime}$ axis; $\odot, B^{\prime}$ axis; $\triangle, D$ axis; $X, C^{\prime}$ axis. (a) $i_{0}=125 \mathrm{ma}, I_{p}=0$; (b) $i_{0}=0, I_{p}=250 \mathrm{ma}$; (c) Potential pattern surrounding the cylindrical couple compared with that around a uniform cylinder of the same size having the same external.current applied.

cylindrical couples measured along the $A$ axes, parts (b), with corresponding space potentials of a copper tube having the same external diameter as the cylindrical couples. External cathodic currents were applied to the copper tube equal to the respective values of $I_{p}$. The ordinates, $\Delta E_{p}$, parts (c), are the algebraic differences obtained by subtracting the copper-tube potentials from the potentials of the respective cylindrical couples. It is noteworthy

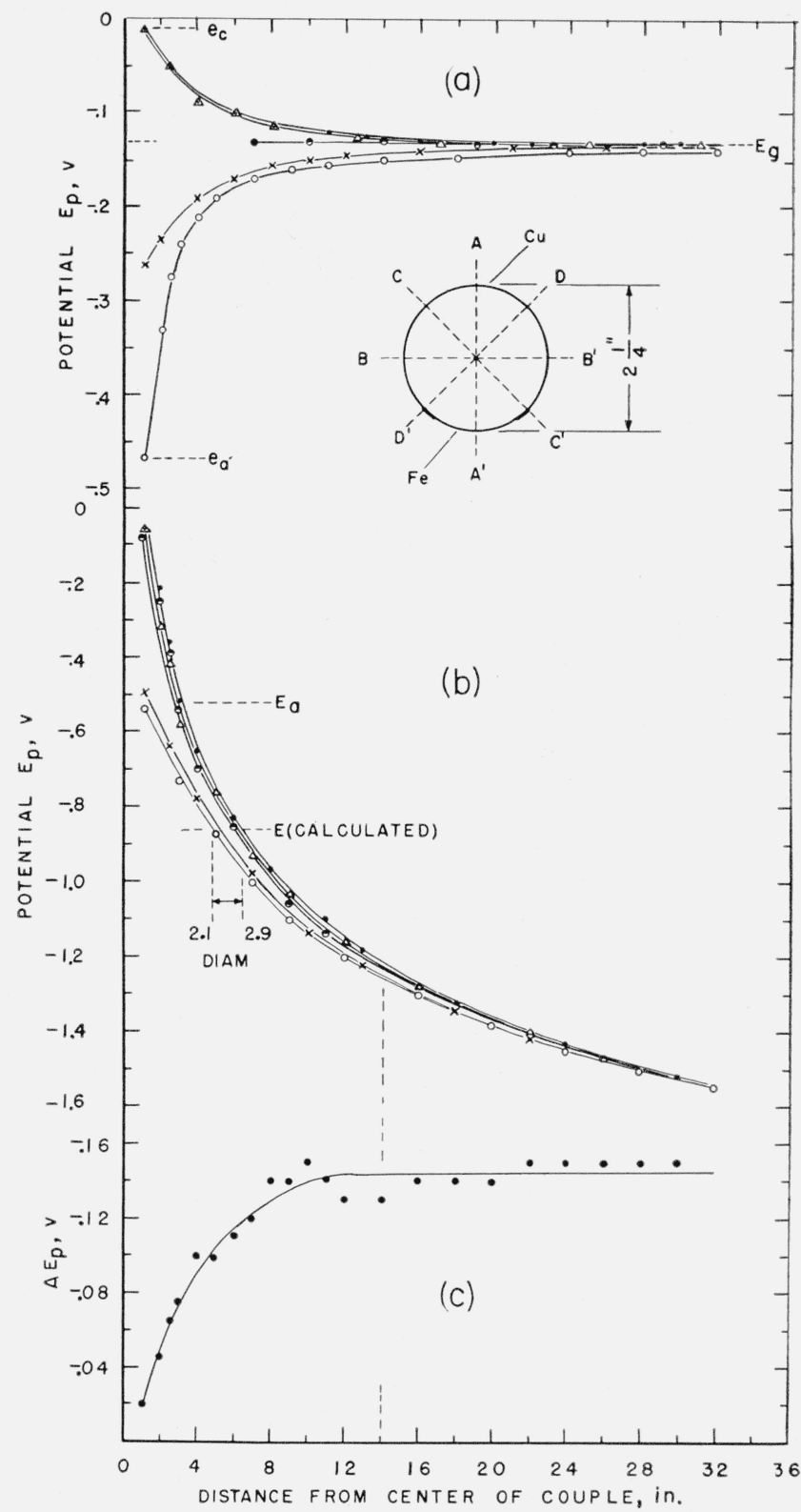

FIgure 10. Cylindrical galvanic couple in a one-tenth normal copper sulfate solution. Area ratio, $\mathrm{Fe}: \mathrm{Cu}=1: 4$.

(a) $i_{0}=110 \mathrm{ma}, I_{p}=0$; (b) $i_{0}=0, I_{p}=420 \mathrm{ma}$; (c) Same as figure 9 (c).

that for distances beyond the effective electrical boundary (14 in.) the effect of the couple becomes relatively constant, therefore indicating that potentials, $E_{p}$, parts (b), beyond 14 inches along any axis vary as the function $\log D / a$ (fig. 3). It will also be noted that beyond 14 inches the ordinates, $\Delta E_{p}$, parts (c), are approximately equal to the couple potentials $E_{g}$, parts (a). 


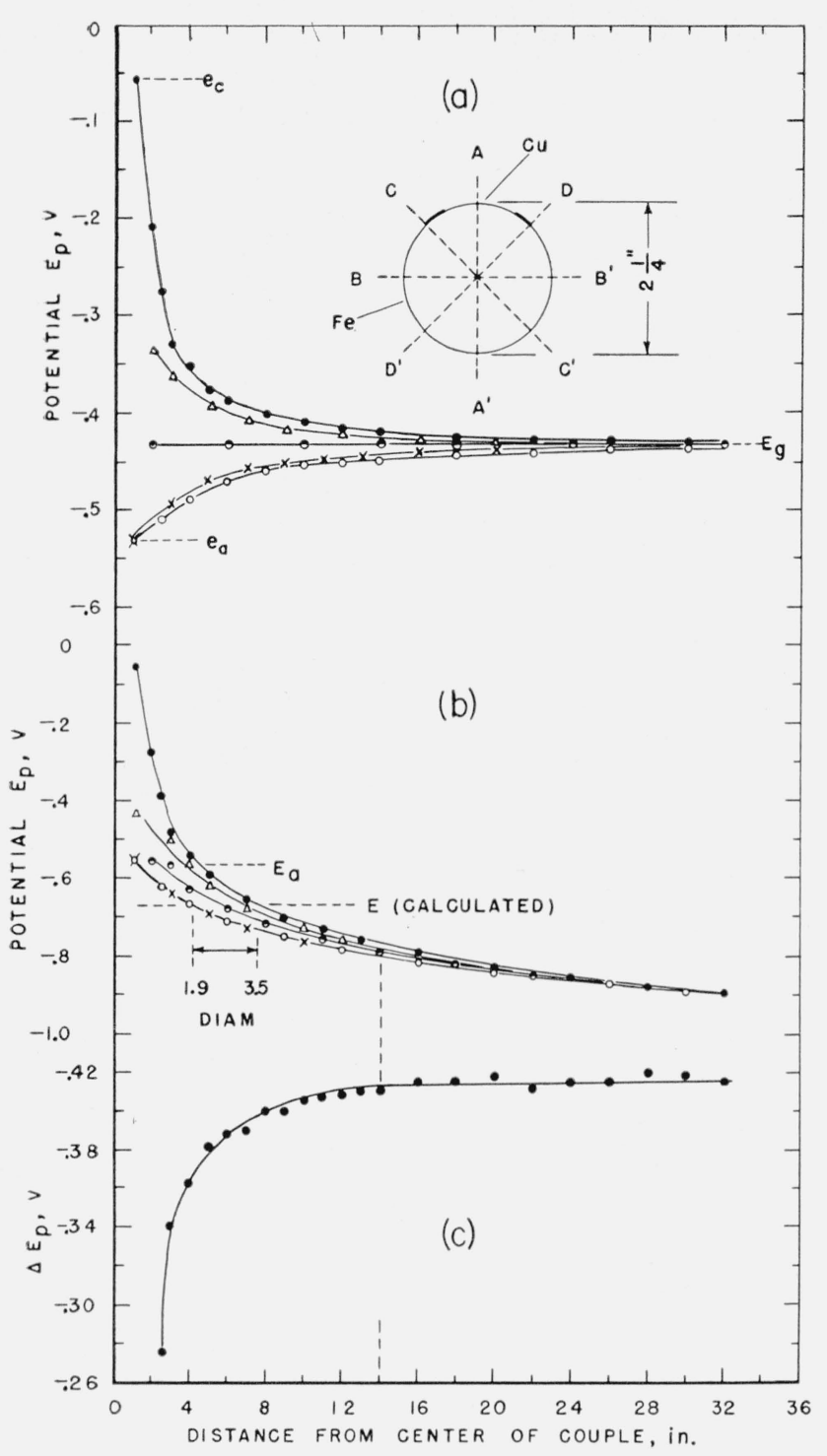

Figure 11. Cylindrical galvanic couple in a one-tenth normal copper sulfate solution.

Area ratio, $\mathrm{Fe}: \mathrm{Cu}=4: 1$

(a) $i_{0}=110 \mathrm{ma}, I_{p}=0$; (b) $i_{0}=0, I_{p}=146 \mathrm{ma}$; (c) Same as figure 9 (c).

\subsection{Galvanic Couples with Electrodes Consisting of Cylindrical Segments in Tap Water}

The experiments carried out with the cylindrical couples exposed to $N / 10$ copper sulfate solution were repeated by exposing the same models to Washington, D. C., tap water (resistivity $=5,200 \mathrm{ohm}-\mathrm{cm}$ at $60^{\circ}$ F) with the idea of observing the effect of polarization, if any, on the electrical boundary. Data for the $1: 1$ couple are shown in figure 12 . That the change in potential, part (a), due to resistance, along the $A A^{\prime}$ axis agrees fairly well with the theoretical change for line electrodes (fig. 2, b) is borne out. For example, at 14 inches the change in potential from the potentials, $e_{a}$ and $e_{c}$, to $E_{g}$ averages about

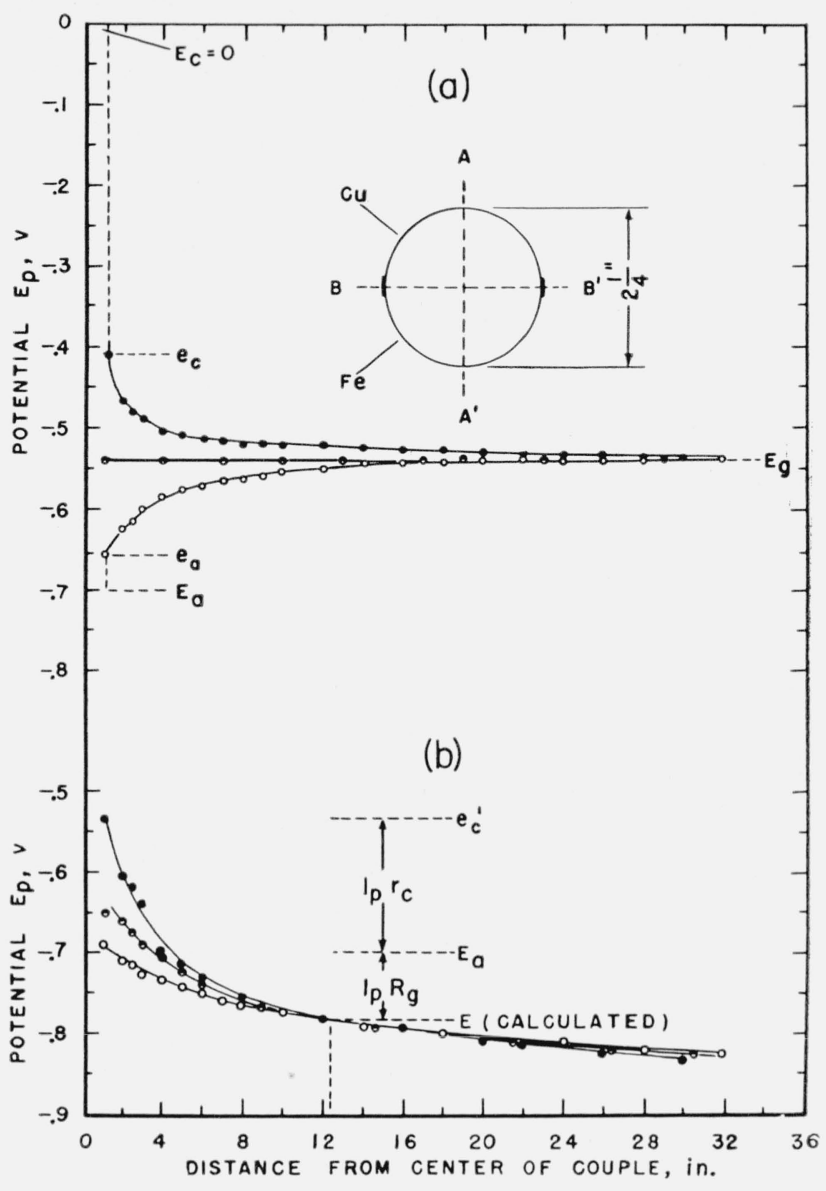

Figure 12. Cylindrical galvanic couple (fig. 9) in Washington, D. C. tap water.

-, $A$ axis; $\bigcirc, A^{\prime}$ axis; $\bullet, B^{\prime}$ axis. (a) $i_{0}=3.5 \mathrm{ma}, I_{p}=0$; (b) $i_{0}=0, I_{p}=4.8 \mathrm{ma}$.

92 percent. When external cathodic current, $I_{p}$, is applied to the couple the curves converge near the calculated boundary potential, which occurs 5.5 diameters from the center of the couple. The divergence of the curves beyond this point is of no significance and is to be disregarded. The calculated boundary potentials for each of the other two models in tap water, geometrically expressed, occurred at 3.5 diameters, averaging therefore 4.2 diameters for the three models. These electrical boundaries are about the same as the values observed in the copper sulfate solution, in which polarization of the electrodes was relatively insignificant as indicated by comparing the potential changes $\left(E_{c}-e_{c}\right)$ in figures 9 and 12 .

As polarization is of major importance in cathodic protection, and the criterion for protection is ordinarily based on a predetermined potential measured between the corroding metal and a reference electrode, the position of the latter must be considered with regard for $I R$ drop included in the measurement. The significance (fig. 12, b) of the potential, $E$, at the electrical boundary as related to the protective potential criterion is discussed in section 4.3. 


\subsection{Effect of Interference}

All experimental data previously discussed were obtained under almost ideal conditions; that is, the tank holding the electrolyte offered little or no interference to the normal flow of current between the anodes and cathodes of the couples. Also, when external current was applied to a vertical cylinder in the center of the tank the current flow was substantially radial, therefore producing nearly equal potential gradients between any two concentric circles. Practical conditions are generally not ideal, and therefore the experiment illustrated by figure 9 was repeated with a vertical insulating wall (fig. 13) positioned inside of the vat, thereby shielding any externally applied current normally flowing from that segment of the auxiliary anode. The normal flow of galvanic current is also affected by the insulating wall.

Prior to obtaining data on the galvanic couple, external cathodic current was applied to a 2.25 -inchoutside-diameter (same as couple diameter) copper pipe positioned vertically in the center of the vat. Potentials measured between the pipe and the insulating wall along the $A A^{\prime}$ axis and also along the insulating wall were found to fit logarithmic equations used by Scott [6] and shown by him to apply to the effect of the earth's cover (represented by the dimension, $h$, fig. 13) on potentials in a plane normal to a pipe line buried parallel to the contour of the earth's surface. Thus, it was established that the Bakelite insulating wall produced interference effects similar to those obtained by Scott, and therefore, the data obtained with the arrangement shown in figure 13 might be considered as being representative of the effect produced by the earth's surface.

Data on one of the couples, with the wall in two positions, are shown plotted in figures 14 and 15 . Included also for comparison are data, parts (a), applicable when the insulating wall is removed. The breaks in the curves, $A$, parts (b and c), mark the distances, $h$, along the $A$ axis to the insulating wall,

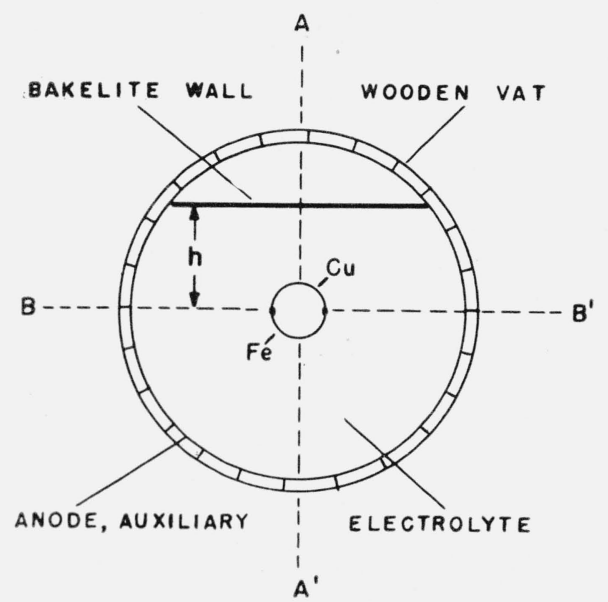

Figure 13. Plan view: Cylindrical galvanic couple (fig. 9) and interference wall (Bakelite) in a one-tenth normal copper sulfate solution.

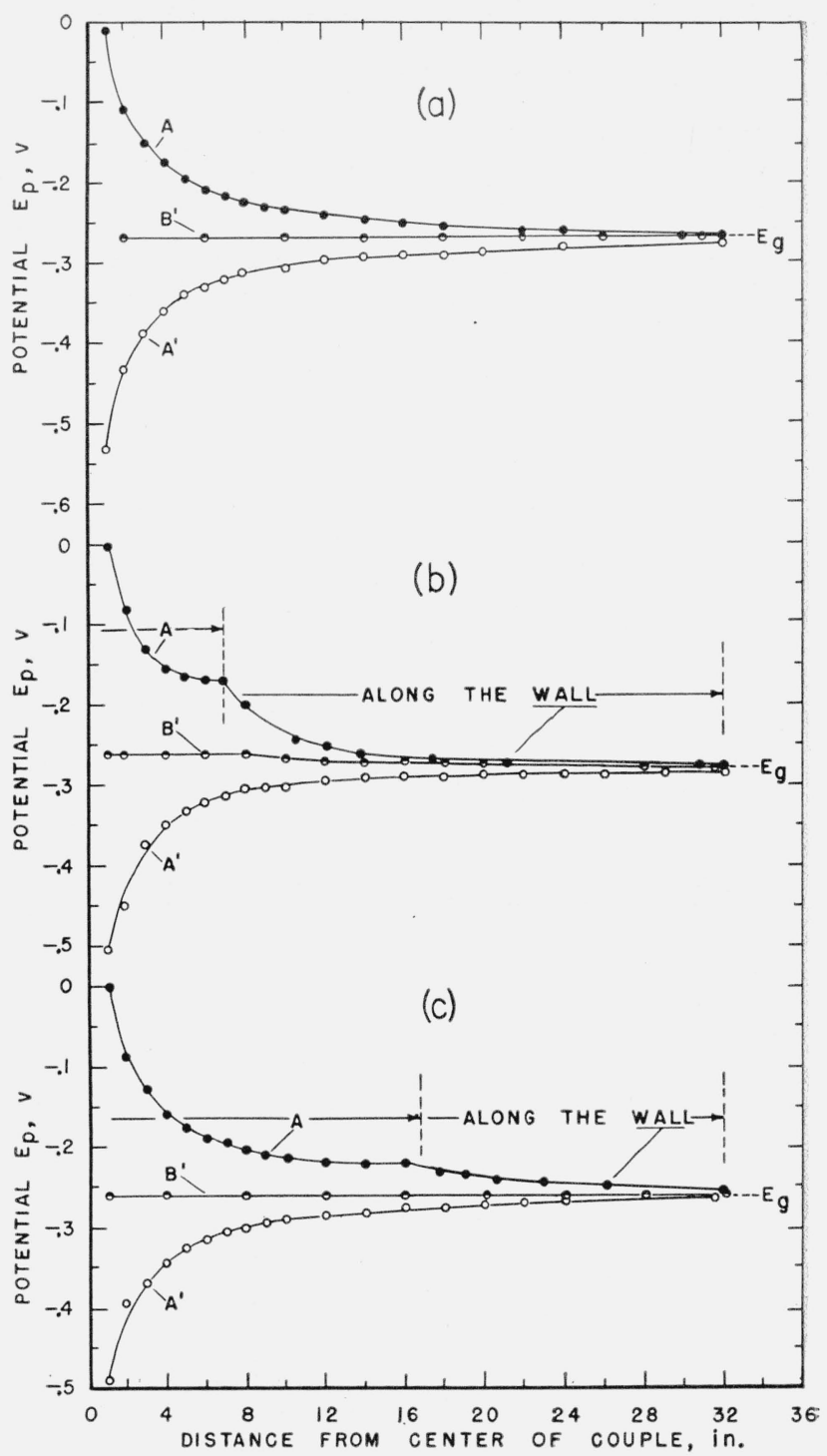

Figure 14. Effect of the interference wall (fig. 13) on the potential lines surrounding the galvanic couple.

(a) No wall, $i_{0}=135 \mathrm{ma}$; (b) With wall at $h=6.85$ in., $i_{0}=135 \mathrm{ma}$; (c) With wall at $h=16.75$ in., $i_{0}=135 \mathrm{ma}$.

after which the radial distances from the couple center pertain to measurements made along the insulating wall. It will be noted that the wall has the effect of reducing the current density and hence the corresponding IR drops along the $A$ axis, as shown by the relative potentials in parts $(a, b$, and c) of the figures. Without external current (fig. 14), the potential along the $B B^{\prime}$ axis remains relatively uuaffected by the insulating wall, and with external current (fig. 15) the disturbance also seems to be a minimum along the $B B^{\prime}$ axis, especially in the range from 3 to 6 diameters. That the insulating wall was really effective as a shield, aside from the data fitting appropriate equations, was the fact that when the external current of 270 ma was applied to the couple, the maximum potential variation in the space between the insulating wall and the shielded. segment of the auxiliary anode was only $20 \mathrm{mv}$. 


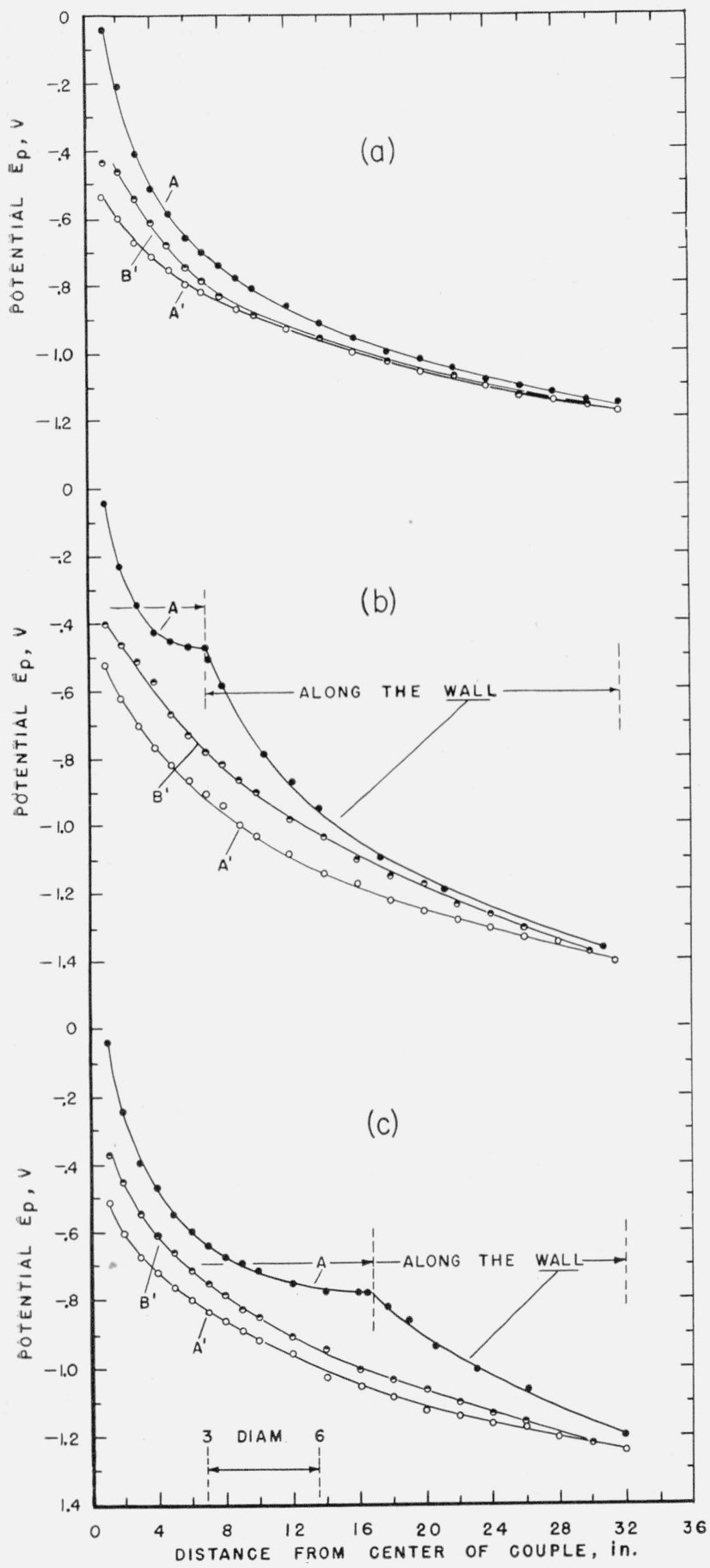

Figure 15. External cathodic current applied to the couple (fig. $14(a))$.

(a) No wall, $i_{0}=0, I_{p}=270 \mathrm{ma}$; (b) With wall at $h=6.85 \mathrm{in}$., $i_{0}=0, I_{p}=270 \mathrm{ma}$ (c) With wall at $h=16.75 \mathrm{in}$., $i_{0}=0, I_{p}=270 \mathrm{ma}$.

\section{Practical Application of the Geometric Relation}

\subsection{General Considerations}

The preceding geometric considerations are applicable in making potential measurements on structures of similar configuration, such as underground horizontal cylindrical tanks and pipelines. In making the application to pipelines it is necessary to assume that most of the corrosion occurring on such lines can be attributed to local action and not to long-line currents. There is good evidence that such is the case. Based on current-flow calculations pertaining to nearly 13 miles of 10 -inch pipeline, Scott [7] concluded that only 6 percent of the accountable total current appeared as long-line current. Scott considered most local action as resulting from current leaving the bottom of the pipe, where the soil is apt to be moist and deficient in oxygen, and entering the top of the line, where the adjacent soil tends to be less moist and more accessible to oxygen. It seems that this would be particularly true for large-diameter pipes where there is a considerable difference in soil cover from the top to the bottom of the pipe and where the weight of the pipeline, causing compression of the underlying soil, is also apt to be a factor. Also, further evidence is based on an analysis of other data, obtained by Scott in conjunction with the American Petroleum Institute pipe-coating tests, by Logan, Ewing, and Denison [8], who found a fair statistical correlation between the corrosion that occurred on bare sections of operating lines and the corrosion of short lengths of small-diameter pipe located nearby in the same trench.

\subsection{Location of Corrosive Areas}

Scott [7] discussed the value of pipe-to-soil potentials in disclosing large anodic or cathodic areas along a pipeline right-of-way and also as a way of predicting the general nature and probable extent of corrosion. He also gave a range of pipe-to-soil potentials for iron pipe as related to aeration of the soil, bacteriological activity, and relative age of the pipe.

Utilities $[9,10,11]$ measure pipe-to-soil potentials to help in the detection of corrosive areas and in order to decide what protective measures should be taken against soil corrosion.

If, on large-diameter pipelines, corrosion circuits are visualized as existing in the geometrical sense, previously discussed, then a pipe-to-soil potential with the reference electrode placed directly over the line would not represent the corrosion or couple potential because the reference electrode would be within the electrical boundary. The error in the reading, assuming normal soil cover, would depend on the soil resistivity and the size of the pipeline. As the corrosion of ferrous materials in soils seems to take place under cathodic control [12], the positioning of the reference electrode as a function of the 
pipe diameter, for example 4 to 6 diameters, would result in the measured potentials being relatively more significant.

\subsection{Cathodic Protection}

According to relatively recent reports of the National Association of Corrosion Engineers and the American Gas Association [13, 14], the pipe-to-soil potential is the chief criterion for evaluating the adequacy of cathodic protection. A reading of these reports leaves one with the impression that the positioning of the reference electrode ought to be done more discriminately. The Corrosion Committee of the AGA revealed that utility companies, applying the $-0.85 \mathrm{v}$ (ref. $\mathrm{Cu}-\mathrm{CuSO}_{4}$ ) protective potential criterion to uncoated pipelines, placed the reference electrode at varying distances from their lines, some placing it directly over the line and others from 5 to 400 feet away. These practices are not conducive to either economical protection for the one extreme or effective protection for the other. The bearing on costs due to such procedures was brought out in a study by Van Nouhuys [15] on 350 miles of 8-inch bare pipeline, wherein it was estimated that in applying the $-0.85-\mathrm{v}$ criterion, the cost for cathodic protection with the reference electrode directly over the pipe was more than four times that figured with the electrode positioned at an extreme distance 300 feet away from the line.

The reason for the apparent differences in the current required for the cathodic protection of a given bare pipeline can be illustrated by the laboratory data (fig. 12, b), in which the boundary potential, $E$, might be thought of as representing the generally accepted protective potential $(-0.85 \mathrm{v})$. The preassigned potential, $E$, ordinarily considered necessary for complete protection, can be observed with the reference electrode at different distances from the center of the couple by changing the value of external current, $I$. For example, to measure the potential, $E$, inside of the electrical boundary (4 to 6 diameters) requires an increase in external current, and outside of the boundary a reduction of current is obviously necessary. The laboratory data might be considered as applying, for example, to the cathodic protection of a long uncoated 12 -inch pipeline receiving protective current from remote ground beds. Potentials should be measured at intervals along the line consistent with usual practices, but with the reference electrode placed from 4 to 6 pipe diameters away. Because of the shielding effect of the earth's surface, the measurements should preferably be made in a horizontal plane through the center of the pipeline or at least in a plane somewhat below the earth's surface. Referring to figure 12 , it will be observed that some allowance, depending on the soil resistivity, should be made for $I_{p} R_{g}$, since the protective potential is actually based on $E_{a}$. It should be pointed out that in other laboratory experiments, not discussed, the value $I_{p} R_{g}$ was found to be negligible for practical purposes when the cylindrical models were exposed to an electrolyte consisting of a solu- tion of sodium chloride in tap water having a resistivity of $500 \mathrm{ohm}-\mathrm{cm}$ or lower.

If, in soils in which $I_{p} R_{g}$ cannot be neglected, potentials are measured at the electrical boundary, the potential $-0.85 \mathrm{v}$ might be satisfactory as a criterion for adjusting the current initially applied for cathodic protection. The condition for complete protection is expressed by eq (11), that is, $E=E_{a}-$ $I_{p} R_{g}$, where (fig. 12)

$$
E_{a}=e_{c}^{1}-I_{p} r_{c} .
$$

Although bright iron in contact with an air-free alkaline soil can have a potential, $E_{a}$, as low as $-0.85 \mathrm{v}$, with respect to a copper-copper sulfate electrode, the average open-circuit potential, $E_{a}$, of the anodes on iron or steel field structures, because of the $\mathrm{pH}$ and aeration of the adjacent soil, is ordinarily considerably less negative than $-0.85 \mathrm{v}$. If, after having applied a protective current from some time, for example, to a pipeline in a moderately high resistivity soil, the measured potentials, $E$, had drifted to values more negative than $-0.85 \mathrm{v}$, then, protection would be indicated [16] and $I_{p} R_{g}$ probably compensated for.

\section{Summary}

The theoretical potential pattern in an electrolyte surrounding a pair of coupled line electrodes having different polarities is compared with the potential configuration around an infinitely long cylinder to which uniformly distributed direct current is flowing. $\mathrm{By}$ superimposing the coupled line electrodes longitudinally on the diameter of the cylinder, the effective electrical boundary applicable to the combination can be expressed geometrically.

The location of the electrical boundary was confirmed experimentally by comparing potentials measured between model galvanic couples and a reference electrode in a surrounding electrolyte with calculated boundary potentials which were based on the measured electrode surface potentials and electrolytic $I R$ drops.

By using electrical insulating shields, the effects of nonuniformly distributed current on the potential patterns around the model galvanic couples were studied. The position of the reference electrode, under such conditions of interference, which resulted in measured potentials having the most significance was arrived at experimentally. The insulating shields simulated the effect of the ground surface on pipelines.

The electrical relations observed with the laboratory models are believed to apply during the normal corrosion and cathodic protection of bare underground pipelines. Therefore, as a result of these experiments, when making potential measurements, for example, pipe-to-soil, during line-conditioning surveys or when applying the protective potential criterion for cathodic protection, it is recommended that the reference electrode be placed 4 to 6 pipe diameters from the line, at some distance below the earth's surface and preferably in an approximately horizontal position with respect to the pipeline. 


\section{References}

[1] Charles Kasper, The theory of the potential and the technical practice of electrodeposition, part 2, Trans. Electrochem. Soc. $7 \%, 365$ (1940).

[2] E. R. Shepard and Henry J. Graeser, Jr., Design of anode systems for eathodic protection of underground and water submerged metallic structures, Corrosion 6, 360 (Nov. 1950)

[3] J. M. Pearson, "Null" methods applied to corrosion measurements, Trans. Electrochem. Soc. 81, 485 (1942).

[4] H. D. Holler, Studies on galvanic couples, part I, J. Electrochem. Soc. 97, 271 (1950).

[5] H. D. Holler, Some electrical relations in galvanic couples, J. Research NBS 45, 373 (1950) RP2148.

[6] Gordon N. Scott, Application of the potential function to problems in eathodic protection, Elec. News 10 (May 1940).

[7] Gordon N. Scott, An aspect of the pipe-to-soil potential and related measurements, NBS Soil Corrosion Conference 1943 (unpublished). Excerpts of this paper appeared in Gas (Feb. 1944).

[8] K. H. Logan, S. P. Ewing, and I. A. Denison, Soil corrosion testing, Symposium on Corrosion Testing Procedures, ASTM (1937).
[9] James R. Cowles, Surveying pipe lines for reconditioning data, using surface potentials, Oil and Gas J. 49, $270(1950)$.

[10] M. C. Callahan, How gulf pipe line locates hot spots on crude-oil lines, Oil and Gas J. 49, 312 (1950).

[11] T. L. Canfield, Surface-potential surveying of bare underground pipe lines, Oil and Gas J. 51, 330 (1952).

[12] W. J. Schwerdtfeger and O. N. MeDorman, Measurement of the corrosion rate of a metal from its polarizing characteristics, J. Electrochem. Soc. 99, 407 (1952).

[13] Report of Correlating Committee on Cathodic Protection, National Association of Corrosion Engineers (July 1951).

[14] Report of the Corrosion Committee (DMC-53-3), Survey of Corrosion Mitigation Practices on Underground Gas Pipes, American Gas Association (April 1953).

[15] H. C. Van Nouhuys, Cathodic protection and high resistivity soil, Corrosion 9, 448 (1953).

[16] W. J. Schwerdtfeger and O. N. McDorman, Potential and current requirements for the cathodic protection of steel in soils, J. Research NBS 4\%, 104 (1951) RP2233.

Washington, September 10, 1954. 